\title{
The influence of differences in gas phase between turbulent and laminar acetylene-oxygen combustion flames on diamond growth
}

\author{
J.J. Schermer, W.A.L.M. Elst, L.J. Giling \\ Research Institute of Materials (RIM), University of Nijmegen, Toernooiveld 1, 6525 ED Nijmegen, Netherlands \\ Received 26 September 1994; accepted in final form 19 January 1995
}

\begin{abstract}
This study describes the deposition of polycrystalline diamond layers on molybdenum substrates, using both laminar and turbulent acetylene-oxygen flames. The use of accurate temperature and flow control systems allowed for a good comparison between diamond layers obtained by a specially designed turbulent burner and a commercially available laminar burner. With constant oxygen flows and temperatures, the deposits obtained with both types of flame in general behave similarly as a function of the applied supersaturation and the position of the substrate in the acetylene feather. Usually continuous diamond layers formed by well-faceted connected crystallites were obtained in the central area of the deposits. These parts of the diamond layers are of good quality, as judged from Raman spectroscopy and their blue band A cathodoluminescence emission. Surprisingly the central area, as obtained by the turbulent flame, is somewhat smaller than from the laminar flame. This, as well as other local differences in growth rate, morphology and impurity incorporation of the deposits, is explained by a model involving the increased mixing rates in the turbulent flame compared with the laminar gas phase.
\end{abstract}

Keywords: Diamond synthesis; Polycrystalline diamond films; Acetylene--oxygen flames; Characterization

\section{Introduction}

In 1988 Hirose and Kondo [1] reported diamond deposition by the use of a laminar acetylene-oxygen flame with a small excess of acetylene. Such a flame is characterized by a well-defined conically shaped flame front [2] which is the activation zone of hydrocarbon radicals necessary for the formation of diamond. Inside this cone the gas mixture is heated up but does not react. Beyond the flame front, $\mathrm{CO}, \mathrm{H}_{2}$, atomic hydrogen and all kinds of hydrocarbons, gradually reacting to $\mathrm{CO}_{2}$ and $\mathrm{H}_{2} \mathrm{O}$ by in diffusion of oxygen from the ambient, form the reducing acctylene feather in which diamond deposition is possible. Outside the feather, in the oxidizing outer flame, diamond will be etched rather than grown.

By now laminar flame deposition is a well-established technique capable of growing diamond with deposition rates exceeding $100 \mu \mathrm{m} \mathrm{h}^{-1}[3,4]$ and quality comparable to that of natural type IIa diamond [4,5]. Its main disadvantage is the limited area of deposition and the relatively large radial inhomogeneity in growth rate, morphology and quality of the grown layers. Using a laminar flame it is possible to obtain deposits with a continuous layer in a circular central area of several millimetres in diameter which is more or less homogeneous in thickness and morphology [6].

Recently, diamond deposition by turbulent flames was reported [7-9]. A turbulent flame is characterized by a loud hissing sound and a blurred flame front which is shorter and more rounded than for a laminar flame [2]. This rounded shape of the flame front could offer possibilities to enlarge the homogeneous central area compared with those obtained by a laminar flame operated at similar conditions. Up to now this aspect has not been examined, but contradictory results were reported concerning the quality and growth rates of diamond deposits obtained with both types of flame. Snail et al. [8] observed an increase in quality but a decrease in growth rate for a diamond layer grown on top of a $\{100\}$-oriented natural diamond substrate by a turbulent flame. In this experiment turbulence was obtained by increasing the total gas flow and the exit opening of the burner compared with the laminar situation [3]. Alers et al. [9] argued that a fair comparison between turbulent and laminar flames is only possible if equal gas flows and burner openings are used. By the addition of a turbulence-inducing step in the burner tip 
design they succeeded in stabilizing a turbulent flame using gas flows for which commercially available burners with the same exit opening are still laminar. Using this burner, with a $0.8 \mathrm{~mm}$ opening, they reported that the polycrystalline diamond deposition rate on molybdenum substrates is twice as high as in a laminar flame under similar conditions.

More recently, an upscaled turbulent burner tip (1.4 mm exit opening) of the same design and a commercially available burner with the same opening were used for homoepitaxial deposition on $\{111\}$ - and $\{100\}$ oriented substrates [10]. No significant differences in deposition rate and morphology were found between the single crystals grown by these turbulent and laminar flames. However, at the same time it was concluded that the positions in the acetylene feathers of both flame types cannot be related to each other owing to the large difference in the gas phase geometry between them. In the present study the influences of this difference in geometry on the growth rate, deposition area and morphological quality of diamond deposits will be determined. For this purpose, polycrystalline diamond layers were grown with both flame types on molybdenum substrates. In the experiments both the influence of different gas phase compositions and variations in the substrate distances to the flame front were investigated.

\section{Experimental}

The experimental arrangement used in this study is comparable to that described in detail in previous work [6]. The deposition temperature $\left(T_{\mathrm{d}}\right)$ as measured with a two-colour infrared pyrometer was kept at $1100^{\circ} \mathrm{C}$ in all experiments by a water vaporizing/cooling system. The mass flows of the high-purity source gases oxygen and acetylene are regulated by mass flow controllers (MFCs). Laminar flames were obtained from a commercially available burner, while turbulent flames were obtained by the use of a CSEM-designed burner [9], both with a $1.4 \mathrm{~mm}$ opening. The turbulent burner was designed for total gas flows of circa $6 \mathrm{slm}$. Because diamond deposition takes place in a narrow composition region around a 1:1 mixture of oxygen and acetylene $[11,12]$, the oxygen flow $\left(f_{\text {ox }}\right)$ during all experiments was kept at $3 \mathrm{slm}$. For these flow rates the Reynolds numbers of the laminar and turbulent flames were calculated to be 2000 and 7000 respectively [10]. The gas phase is determined by $f_{\text {ox }}$ and the acetylenc supcrsaturation $\left(S_{\mathrm{ac}}\right)$. At a constant oxygen flow, $S_{\mathrm{ac}}$ is defined as the actual acetylene flow minus the acetylene flow of the ideal welding flame divided by the latter. The ideal welding flame, for which the acetylene feather has just disappeared, is determined by sight. The distance $(d)$ between the substrate and the flame front (the activation zone) is determined by a cathedometer. The fact that the flame front of the turbulent flame is ill defined obstructs the accurate determination of $d$ and the ideal welding flame which determines the zero point in $S_{\mathrm{ac}}$. This problem is solved by using the substrate to burner distance $(b)$ and the acetylene to oxygen ratio $(R)$ in a turbulent flame as an alternative reference for $d$ and $S_{\mathrm{ac}}$ respectively. Before a growth experiment with the laminar flame the ideal welding flame was determined and the gas flows installed according to the desired $S_{\mathrm{ac}}$. Each of these laminar experiments was directly followed by its turbulent counterpart using the same gas ratio $R$. The conditions which were kept constant in the experiments as well as the ranges in which the investigated parameters were varied are given in Table 1.

In this study commercially available $99.6 \%$ pure acetylene was used, which was dissolved in acetone in the gas bottle. Therefore the acetylene flow always contained a certain acetone fraction. This fraction increases with decreasing pressure and increasing temperature in the gas cylinder. The acetone fraction, which in addition influences the heat capacity-dependent regulation of the MFCs in the acetylene line, determines the gas composi-

Table 1

Deposition conditions (see text for symbols)

Fixed deposition conditions for all experiments

Burner tip, exit opening

Substrate

Deposition time

Deposition temperature $\left(T_{\mathrm{d}}\right)$

Oxygen flow $\left(f_{\text {ox }}\right)$
Laminar: Standard design, $1.4 \mathrm{~mm}$ dia.; Turbulent: CSEM design, $1.4 \mathrm{~mm}$ dia.

$12 \times 12 \times 0.5 \mathrm{~mm}^{3}$ molybdenum

$1 \mathrm{~h}$

$1100{ }^{\circ} \mathrm{C}$

$3.0 \mathrm{sIm}$

Series of experiments with varied deposition conditions

\begin{tabular}{llll}
\hline Fixed & Parameter & Range & Fig. \\
\hline$d=1.5 \mathrm{~mm}$ & $S_{\mathrm{ac}}(\%)$ & $1.5-10(R=1.09-1.17)$ & 2,3 and 4 \\
$S_{\mathrm{ac}}=5 \%$ & $d(\mathrm{~mm})$ & $0.4-2.8(b=4.9-7.3)$ & $5(\mathrm{~b}), 7(\mathrm{a})$ and $7(\mathrm{~b})$ \\
$S_{\mathrm{ac}}=5 \%$ & $d(\mathrm{~mm})$ & $1.0-10.0(b=5.5-14.5)$ & $5(\mathrm{a}), 6,7(\mathrm{c})$ and $7(\mathrm{~d})$ \\
\hline
\end{tabular}


tion and the released combustion energy. Finally, this changed the diamond deposition process by a decrease in temperature at the flame front and the carbon to oxygen ratio in the feather. Part of this effect was compensated by the determination of the ideal welding flame and using $S_{\mathrm{ac}}$ as a reference for the gas composition. Note that, therefore, within a series of experiments $S_{\mathrm{ac}}$ and $R$, as determined by the read-out of the MFCs, in general does not have a linear relation. In addition, the influence of this effect on the growth results was minimized by starting each series of experiments with a new gas bottle using only the first part of the content. Due to differences in the bottle content this procedure may lead to small deviations between the series, but within a series of experiments the results are consistent [6].

Square $12 \times 12 \mathrm{~mm}^{2}$ molybdenum substrates with a thickness of $0.5 \mathrm{~mm}$ were used in all experiments. To ensure good thermal contact with the cooling system these substrates were soldered onto the substrate holder using a high-temperature soldering alloy. Prior to deposition the substrates were scratched with sandpaper and micron-sized natural diamond powder to enhance diamond nucleation. Immediately before growth the scratched substrates were cleaned with isopropanol, blown dry in air and etched for $1 \mathrm{~min}$ in the oxidizing outer flame. A deposition time of $1 \mathrm{~h}$ was used for all experiments.

The large-scale morphology of the grown samples was investigated by a binocular microscope (BM). The morphology of the deposits was examined in detail by optical differential interference contrast microscopy (DICM) and scanning electron microscopy (SEM, type JEOL JSM-T300). In addition, the optical microscope was used to determine the thickness of the diamond layers in the central area [6]. Raman spectroscopy was employed to analyse the quality of the grown material, and the spatial distribution of luminescent point defects was analysed by cathodoluminescence topography (CL). Both the Raman and CL setup have been described in previous work $[4,13]$.

\section{Results}

For the sake of convenience the diamond layers grown by the turbulent flame and the laminar flame will be referred to as turbulent samples and laminar samples respectively. Most of the turbulent as well as the laminar samples which are discussed in the present study are circular symmetric, as shown in Fig. 1. In the centre the deposits usually form a continuous layer of connected randomly oriented diamond crystallites with $\{111\}$ as well as $\{100\}$ facets. Most of these crystallites are twinned and show secondary nucleation. The central area is surrounded by an annulus revealing a low density

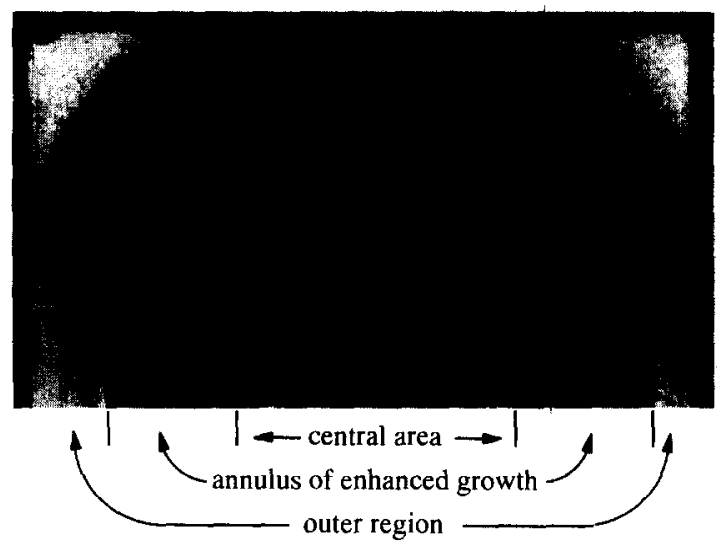

Fig. 1. BM image of somewhat more than half of a turbulent sample $\left(S_{\mathrm{ac}}=5 \%\right.$ and $d=1.6 \mathrm{~mm}$ ) showing the typical large-scale morphology observed for most of the deposits obtained by turbulent as well as laminar flames. The regions with green, yellow, brown, orange and blue emission as observed by $C L$ topography are indicated by the first letters of the colours. The dimension of the sample in the horizontal direction is $12 \mathrm{~mm}$.

of large columnar crystallites separated by voids or embedded in an amorphous layer of ball-like features. These crystallites are without distinct side faces but quite often have $\{100\}$ top faces more or less parallel to the substrate. Beyond this annulus the deposits have the same structure as the central region, with a decreasing size of the crystallites radially away from the centre. A similar radially changing structure, although with a central area dominated by $\{100\}$ faces, has been reported by Ravi [14]. In a previous study [6] the occurrence of these $\{100\}$ faces in the central area was found to be enhanced by increasing temperature, increasing supersaturation and decreasing flow rate. However, it is evident that only the continuous central area within the annulus of isolated crystallites of the deposits has a growth rate and morphology which makes it suited for practical use in future applications. Therefore, unless stated otherwise, this zone, which also exhibits the highest quality (see next section), is considered in the present study.

\subsection{Cathodoluminescence topography}

Inspection by $\mathrm{CL}$ topography of the pre-treated substrates directly before growth, showed a homogeneously distributed bright blue emission, which was not observed on the untreated substrate and after scratching with sandpaper. This blue band A luminescence from donoracceptor pairs [15], which is characteristic for natural diamond, shows that scratching with diamond powder enhances nucleation not only by creation of new deposition sites at the scratches [16], but also by the remaining diamond seed particles [17]. These particles are embedded relatively tightly in the molybdenum substrates since they were not removed by the isopropanol cleaning procedure. 
After growth CL observations of both the turbulent and the laminar samples reveal a radially changing luminescent behaviour of the diamond deposits, as indicated in Fig. 1. In this section a general description of this behaviour is given, which is valid for most of the samples discussed in this study. The central areas usually reveal blue band A luminescence, with a very small ring of orange-red luminescence at their outmost borders. This orange-red $575 \mathrm{~nm}$ system indicates the incorporation of nitrogen as nitrogen-vacancy pairs in the diamond lattice $[18,19]$. The annulus around this area, which contains only a limited amount of crystallites with $\{100\}$ top faces, does not reveal any luminescence. Beyond this annulus, the luminescence starts off with orange again and gradually changes via brownish and yellow to green at the outmost boundary of the deposits.

These CL observations provide so much information that it is possible to give a direct explanation for the peculiar morphology of the diamond deposits as described above. During deposition, $\mathrm{N}_{2}$ diffuses into the acetylene feather, decomposes by thermal dissociation or by reactions with other species and can be built into the diamond lattice. In the gas phase above the outer boundary of the deposits the nitrogen is only partly decomposed. Consequently, the incorporation into the diamond lattice is relatively low, resulting in a green CL signal obtained by the combined blue band A luminescence and emission from the $575 \mathrm{~nm}$ system. Towards the centre of the acetylene feather the decomposition of $\mathrm{N}_{2}$ increases, leading to a larger incorporation in the diamond lattice and shifting of the CL signal via yellow and brownish towards orange-red of the $575 \mathrm{~nm}$ system. Directly above the annulus of large isolated crystallites the amount of reactive nitrogen in the gas phase reaches a maximum. The large amount of incorporated nitrogen probably results in a quenching of the CL signal and a dramatic change in the morphology of the deposited layers. The introduction of nitrogen into the gas phase with a nitrogen over carbon ratio above 0.1 was reported to increase the diamond growth rates by more than a factor of $2[20,21]$. Recently we obtained the same results using nitrogen concentrations below 100 p.p.m. in a bot-filament reactor [22]. At the same time Jin and Moustakas [20] reported that growth via the $\{111\}$ faces is obstructed due to the large distortion by about $36 \%$ of the $\mathrm{C}-\mathrm{N}$ bond length towards the $\langle 111\rangle$ directions [23]. These combined effects of nitrogen incorporation can account for the enhanced growth of individual columnar crystallites without $\{111\}$ facets [22]. The maintained presence of $\{100\}$ faces on these crystallites is probably due to stabilization by the formation of dimer bonds between the surface atoms $[24,25]$. The diffusion of nitrogen further into the feather and/or its dissociation apparently decreascs fast, resulting in the "normal" morphology and the dominant blue band A luminescence observed in the central area of the deposits.

\subsection{Deposition with different supersaturations}

The deposition rate $\left(v_{\mathrm{d}}\right)$ as a function of the acetylene supersaturation is given in Fig. 2. The acetylene/oxygen ratio $R$ used in the experiments is indicated on the top

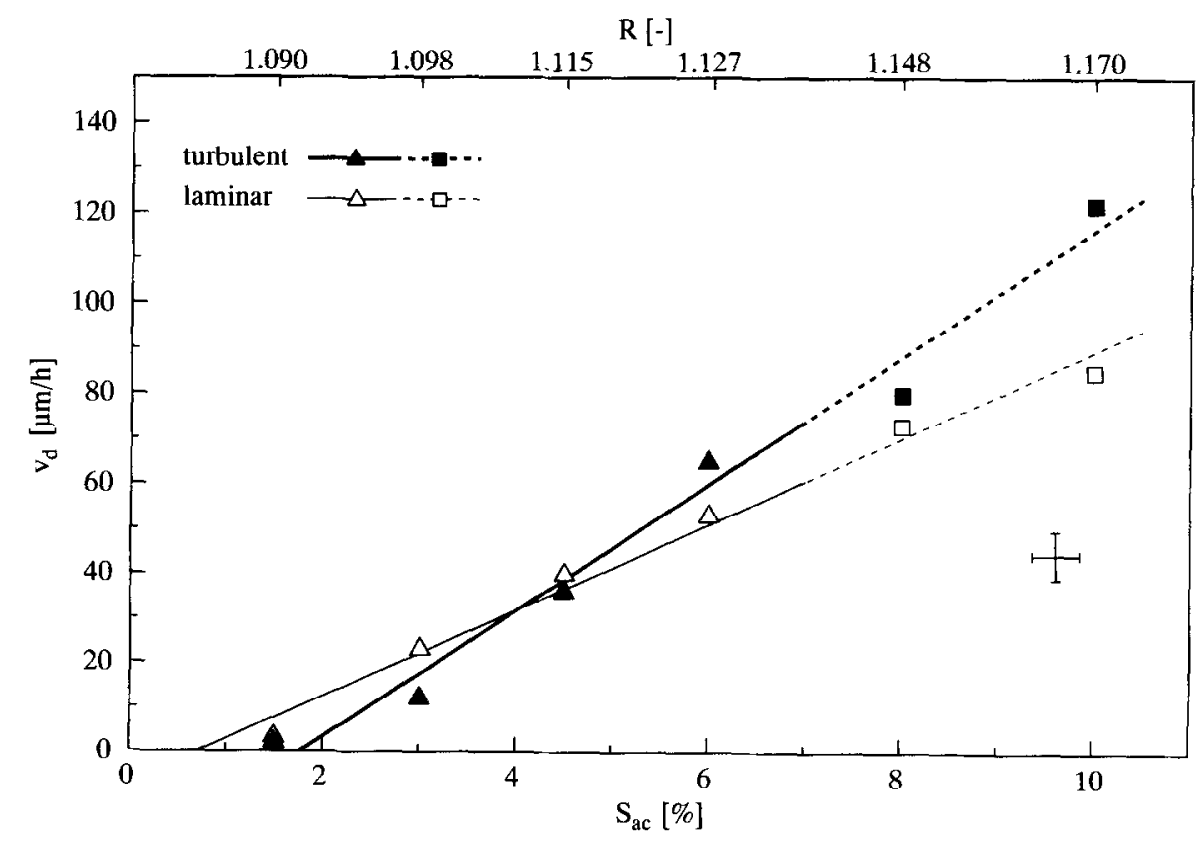

Fig. 2. Deposition rates in the central arcas of diamond deposits obtained by turbulent and laminar flames as a function of the acetylene supersaturation at $d=1.5 \mathrm{~mm}$. Triangles indicate a mixed morphology with $\{111\}$ and $\{100\}$ faces, while squares indicate the dominant appearance of $\{100\}$ faces. The full and broken lines respectively indicate whether the central area of the deposits forms a continuous layer or consists of separate crystallites. The average error in the data is indicated by a representative error bar. 
axis of the figure. During the experiments the substrates were positioned $1.5 \mathrm{~mm}$ below the flame front, which for the turbulent flame only could be estimated within $\pm 0.4 \mathrm{~mm}$. However, by the use of the burner tip as a reference, the positions of the substrates in the turbulent feather could at least be kept the same in each experiment $(b=6 \mathrm{~mm})$. Fig. 2 shows that the growth rate is approximately linear with $S_{\text {ac }}$ for both flame types. The best linear fits through the data points show that the deposition rate of turbulent layers increases faster with $S_{\mathrm{ac}}$ (or $R$ ) than that of the laminar specimen. Although the range of the gas phase compositions investigated was much smaller than in the present study, the results reported by Alers et al. [9] when extrapolated also show the crossing of lines representing the laminar and turbulent data. Both lines in Fig. 2 cross the positive $S_{a c}$-axis because for low supersaturations the acetylene feathers become too small to have an intersection with the substrate at $1.5 \mathrm{~mm}$ distance from the flame front. The line representing the laminar data crosses closer to the origin, since the laminar feather is longer [10]. The broken lines in Fig. 2 indicate that for $S_{\mathrm{ac}} \geqslant 8 \%$ the central area of the deposits does not form a continuous layer. The shape of the markers is representative for the morphology of the individual crystallites of the layer, as discussed below.

Surprisingly the central area of the samples grown by the turbulent flame is smaller and the width of the annulus around it is slightly larger compared with the laminar samples. The diameter of the central area as a function of $S_{\mathrm{ac}}$ appears to be fairly constant around $5 \mathrm{~mm}$ for $S_{\mathrm{ac}} \geqslant 3 \%$. Only for $S_{\mathrm{ac}}=1.5 \%$ does this diameter rapidly decrease to $1.5 \mathrm{~mm}$ for the laminar flame, and the central area vanishes for the turbulent flame.

SEM and DICM observations reveal that the morphologies of the turbulent and laminar specimen are very much the same but change dramatically with supersaturation as can be seen in Fig. 3. The central area of samples grown at $S_{\mathrm{ac}}=1.5 \%$ is formed by crystallites generally smaller than $2 \mu \mathrm{m}$, which especially on the turbulent sample are hardly recognizable. Fig. 3(a) shows that layers obtained with $S_{\mathrm{ac}}=3 \%$ are formed by wellfaceted diamond crystallites which, compared with laminar specimen, are significantly smaller on the turbulent layer. The dimensions of the crystallites increase and become equally sized on the samples grown at $S_{\mathrm{ac}}=$ $4.5 \%$ with both flame types (see Fig. 3(b)). Fig. 3(c) reveals that the crystallites grown using $S_{\mathrm{ac}}=6 \%$ are twinned and show a large amount of secondary nucleation, especially in re-entrant corners between the individual crystallites. For higher supersaturations the central area of the deposits does not form a continuous layer but shows individual crystallites with smooth $\{100\}$ top faces (see Fig. 3(d)). Although the morphology of these central areas is very similar to that observed in the annulus around the central area, the density of the
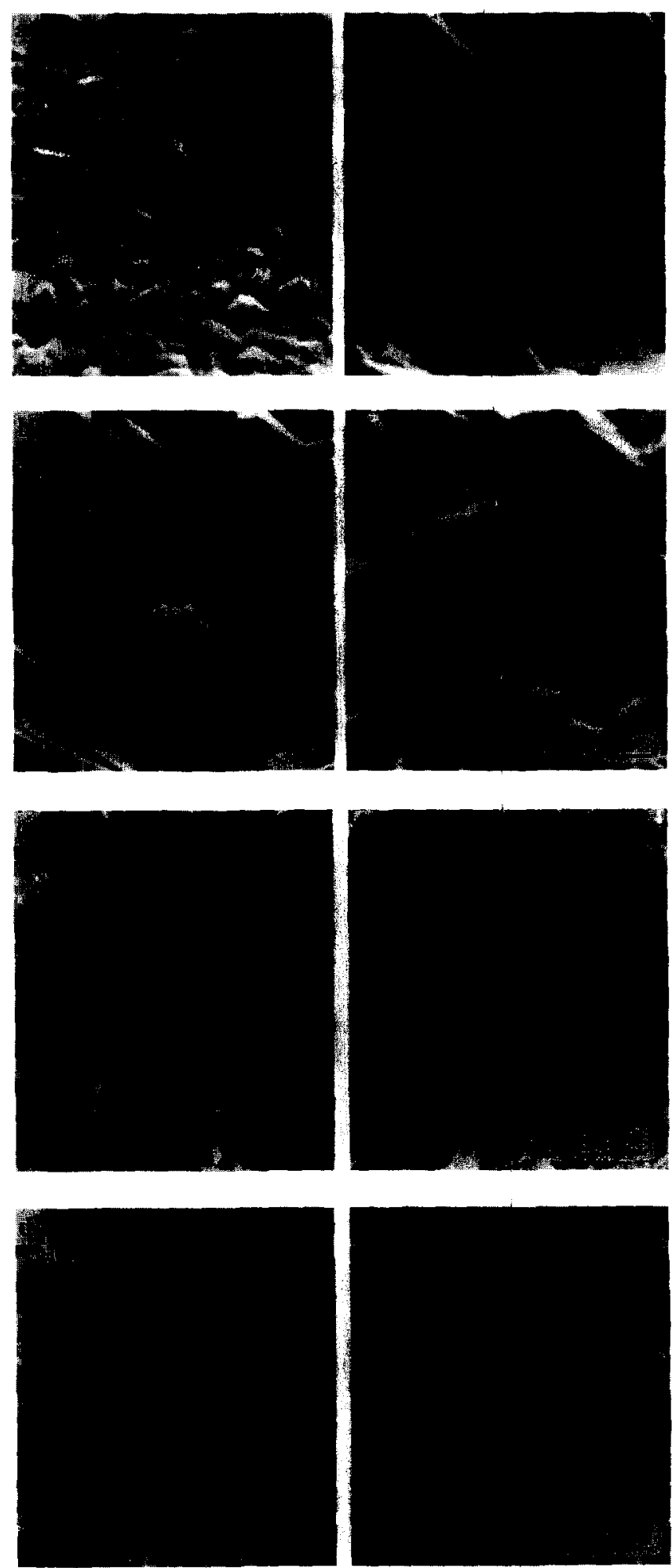

Fig. 3. SEM images showing the detailed morphology of the central areas of turbulent (left-hand side) and laminar (tight-hand side) diamond deposits grown at supersaturations of (a) $3 \%$, (b) $4.5 \%$, (c) $6 \%$ and (d) $10 \%$. Photograph (c) was taken perpendicular to the sample, the others were taken at an angle of $55^{\circ}$ to the normal to the surface. Left-and right-hand sides of the photographs are on the same scale. 
crystallites is much higher and the CL signal is still more or less blue. Therefore it can be assumed that the change in morphology of the central area in this case is not induced by enhanced incorporation of nitrogen but rather by competitive shadowing and nutrient starvation of the crystallites [14]. The occurrence of defects like stacking faults, graphite-like inclusions and microtwinning for diamond crystals grown via $\{111\}$, which are known to increase with the supersaturation [26], resulted in the total deterioration of these faces. Both samples grown at $S_{\mathrm{ac}}=8 \%$ (not shown) and the turbulent sample grown at $S_{\mathrm{ac}}=10 \%$ have a similar appearance to crystallites of approximately the same dimensions. Fig. 3(d) shows that the crystallites on the sample grown at $S_{\mathrm{ac}}=10 \%$ in the laminar flame are significantly smaller. Layers obtained with $S_{\text {ac }}>10 \%$ do not reveal a crystalline structure and are considered to be diamondlike carbon (DLC) [27]. The described differences in size of the crystallites between the turbulent and laminar samples as a function of $S_{\mathrm{ac}}$ support the observed crossing of lines representing the turbulent and laminar growth rates, as shown in Fig. 2.

Fig. 4 shows that the Raman spectra, as recorded between $1100 \mathrm{~cm}^{-1}$ and $1700 \mathrm{~cm}^{-1}$, of the turbulent and laminar samples are quite comparable. Close examination reveals that, relative to the background, the diamond phonon line of the turbulent samples is usually slightly higher. Possibly this is related to an increased etching of graphite by "leaking" of oxygen through the turbulent flame front, as reported in the literature $[5,28]$.
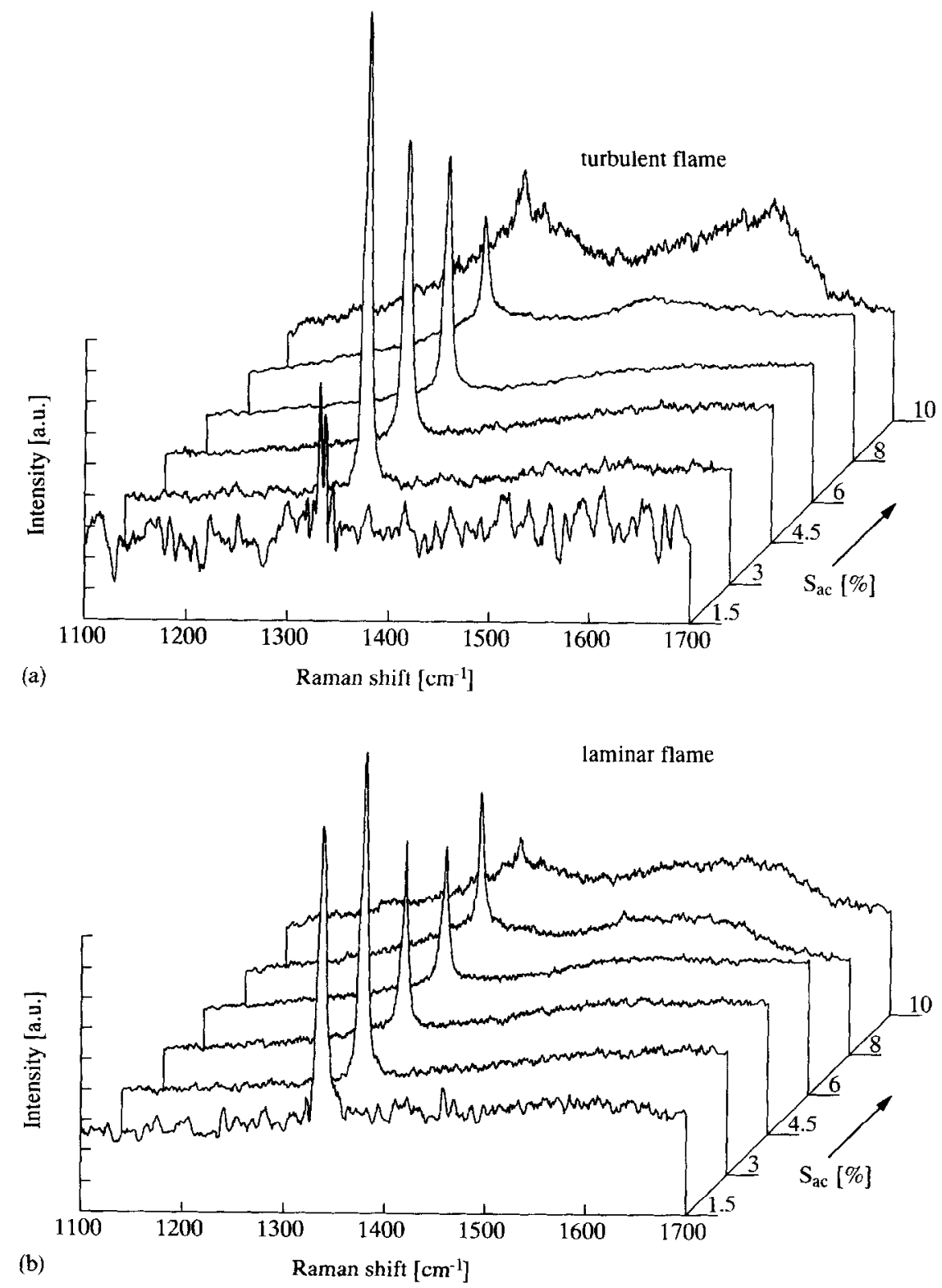

Fig. 4. Raman spectra from the central areas of polycrystalline diamond layers grown by (a) turbulent and (b) laminar flames using different supersaturations. 
There appears to be a close relation between the results of the Raman analysis and the morphology of the samples. The samples grown at $S_{\mathrm{ac}}=1.5 \%$, especially turbulent one, give only just enough signal to prove the presence of any diamond. The spectra of all specimens grown with $3 \% \leqslant S_{\mathrm{ac}} \leqslant 8 \%$ show a clear diamond peak above a luminescent background. Relative to the diamond peak this background increases with supersaturation, especially at higher wavenumbers. Fig. 4 shows that, related to the change in morphology, for samples grown at $S_{\mathrm{ac}}=8 \%$ the broad band of the non-diamond carbon phases between $1400 \mathrm{~cm}^{-1}$ and $1600 \mathrm{~cm}^{-1}$ becomes apparent. This band strongly increases for samples grown at $S_{\mathrm{ac}}=10 \%$ and instead of a sharp diamond peak these spectra exhibit a broad band around $1330 \mathrm{~cm}^{-1}$. For most samples the position of the diamond peak is shifted from $1332 \mathrm{~cm}^{-1}$ towards higher wavenumbers (see Table 2). The fact that free-standing diamond layers, grown under identical conditions in our laboratory, do not reveal this shift indicates that this phenomenon is related to stress due to the difference in expansion between the molybdenum substrates and the diamond layers. During diamond deposition at $1100^{\circ} \mathrm{C}$ the crystallites form a solid connection with the substrate, possibly by intermediate molybdenum carbide layers $[27,29]$. Upon cooling down to room temperature the size of the substrate decreases faster than that of the deposited diamond film. If the deposit forms a continuous layer the crystallites are squeezed together and their diamond lattices are deformed by this compressive stress, resulting in a shift of the diamond peak towards higher wavenumbers $[30,31]$. The diamond phonon lines are not or are significantly less shifted for the samples grown at $S_{\mathrm{ac}} \geqslant 8 \%$ and the turbulent layer grown at $1.5 \%$ because they do not form a continuous layer, and the relative shrinking of the substrates is absorbed by the voids between the crystallites. The highest shift is obtained for the most perfect crystalline layers grown at $S_{\mathrm{ac}}=3 \%$. For $S_{\mathrm{ac}}=4.5 \%$ or $6 \%$ the shifts are less since the strain is partly released by the increasing amount of non-carbon phases between the grain boundaries or the increasing thickness of the layers.

Table 2

Diamond peak position in the Raman spectrum for different supersaturations

\begin{tabular}{lll}
$\begin{array}{l}\text { Acetylene } \\
\text { supersaturation }(\%)\end{array}$ & $\begin{array}{l}\text { Position for } \\
\text { turbulent deposits } \\
\left(\mathrm{cm}^{-1}\right)\end{array}$ & $\begin{array}{l}\text { Position for } \\
\text { laminar deposits } \\
\left(\mathrm{cm}^{-1}\right)\end{array}$ \\
\hline 1.5 & 1333.0 & 1337.7 \\
3.0 & 1339.4 & 1340.0 \\
4.5 & 1338.9 & 1339.0 \\
6.0 & 1338.2 & 1338.5 \\
8.0 & 1333.6 & 1332.1 \\
10.0 & 1332.4 & 1332.0 \\
\hline
\end{tabular}

In order to obtain a closed central area with a maximum diameter and the highest growth rate a supersaturation of $6 \%$ appears optimal for both flame types at the given conditions. If high-quality diamond with well-shaped faces is required, e.g. for the growth of highquality single crystals as frequently employed in our laboratories $[4,10,32], 4.5 \%$ appears more appropriate.

\subsection{Deposition at different distances from the flame front}

For $S_{\mathrm{ac}}=5 \%$ the deposition rate as a function of the distance over the entire length of the feather of the turbulent as well as the laminar flame is given in Fig. 5(a). As stated before, the flame front for the turbulent flame is ill defined; therefore the installed distances of the substrate to the flame front may not exactly correspond to those applied for the laminar flame. However, by the use of the burner tip as a reference the intervals between the different values are the same for both flame types. Fig. 5(a) shows that the growth rate obtained with the turbulent flame initially is constant, but starts to decrease with distance at approximately $4 \mathrm{~mm}$ from the flame front. In contrast with this, close to the flame front the growth rate in the laminar flame decreases fast with increasing $d$ but soon becomes constant. However, as is indicated by the broken lines, most deposits do not form a continuous diamond layer. Therefore a second series of growth experiments was performed under the same growth conditions in the region closer to the flame front. For this series the deposition rates are very similar for both types of flame, although they decrease more gradually with the distance from the flame front for the turbulent layers (Fig. $5(\mathrm{~b})$ ). The broken lines in Fig. 5(b) indicate that for samples grown close to the flame front the central layer starts to break up into large individual crystallites [6]. The use of another acetylene bottle probably accounts for the slightly higher growth rates obtained in the second series compared with the corresponding experiments in the first series. However, the differences are small compared with the absolute inaccuracy in the determination of the growth rate, as indicated by a representative error bar in Fig. 5. Furthermore, the morphologies of the samples grown with the same flame type at the same distance in both series are nearly identical. Therefore the results obtained in the two series will be considered simultaneously in this study and only differences arising from the use of different flame types will be discussed.

As was also observed for the series of experiments with different supersaturations, the central area of turbulent layers is smaller and the width of the annulus around it is slightly larger compared with the laminar layers (Fig. 6). The maximum diameter of the central area is a little larger than $5 \mathrm{~mm}$ for both types of flame and is obtained for $d=0.4 \mathrm{~mm}$. This area decreases slowly with increasing distances until at $d \sim 1.6 \mathrm{~mm}$ this 

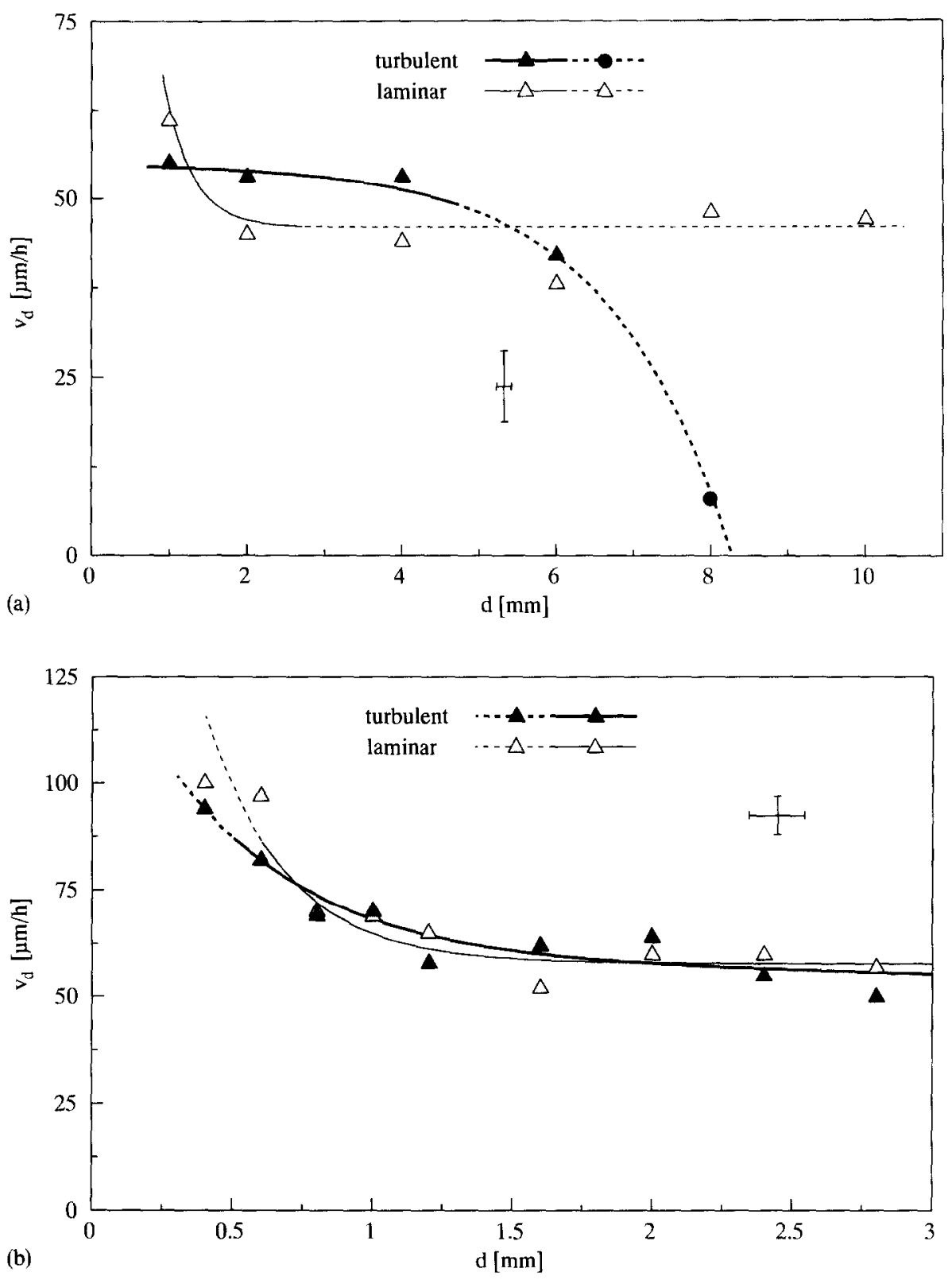

Fig. 5. Deposition rates in the central areas of diamond deposits obtained by turbulent and laminar flames as a function of the distance $d$ of the substrate to the flame front. Experiments at large intervals over the entire length of the acetylene feather (a) and at small intervals in the region close to the flame front (b). The shapes of the lines and triangle markers indicate the same features as stated in Fig. 2; the circular marker indicates that the central area of the deposit is fully covered by an amorphous carbon layer.

area on turbulent specimens starts decreasing rapidly and vanishes for $d=8 \mathrm{~mm}$ (see left-hand side of Fig. 6). For laminar samples the slow decrease of the central area with $d$ is maintained somewhat longer, but an additional zone, to be referred to as the core zone hereafter, arises in the middle of the central areas for $d \geqslant 2.4 \mathrm{~mm}$ (see right-hand side of Fig. 6). For $d<2.4 \mathrm{~mm}$ the central area of the laminar samples was already observed to be slightly less homogeneous than the turbulent samples. At this point it should be noted that the growth rates shown in Fig. 5 are related to the deposit just outside this core zone. Furthermore, in contrast with the turbulent specimen, the central areas of the laminar samples become irregularly shaped at larger distances, indicating lower stability of the laminar flame.

Close observations by DICM and SEM reveal that the samples grown close to the flame front have the general morphology as described at the beginning of Section 3 and which is shown in Figs. 3(b), 3(c) and the left-hand sides of Figs. 7(a) and (b). Further away from the flame front the morphology of the central area on turbulent samples gradually changes (see left-hand side of Fig. 7). For $d=4 \mathrm{~mm}$ the crystallites in the layer start 

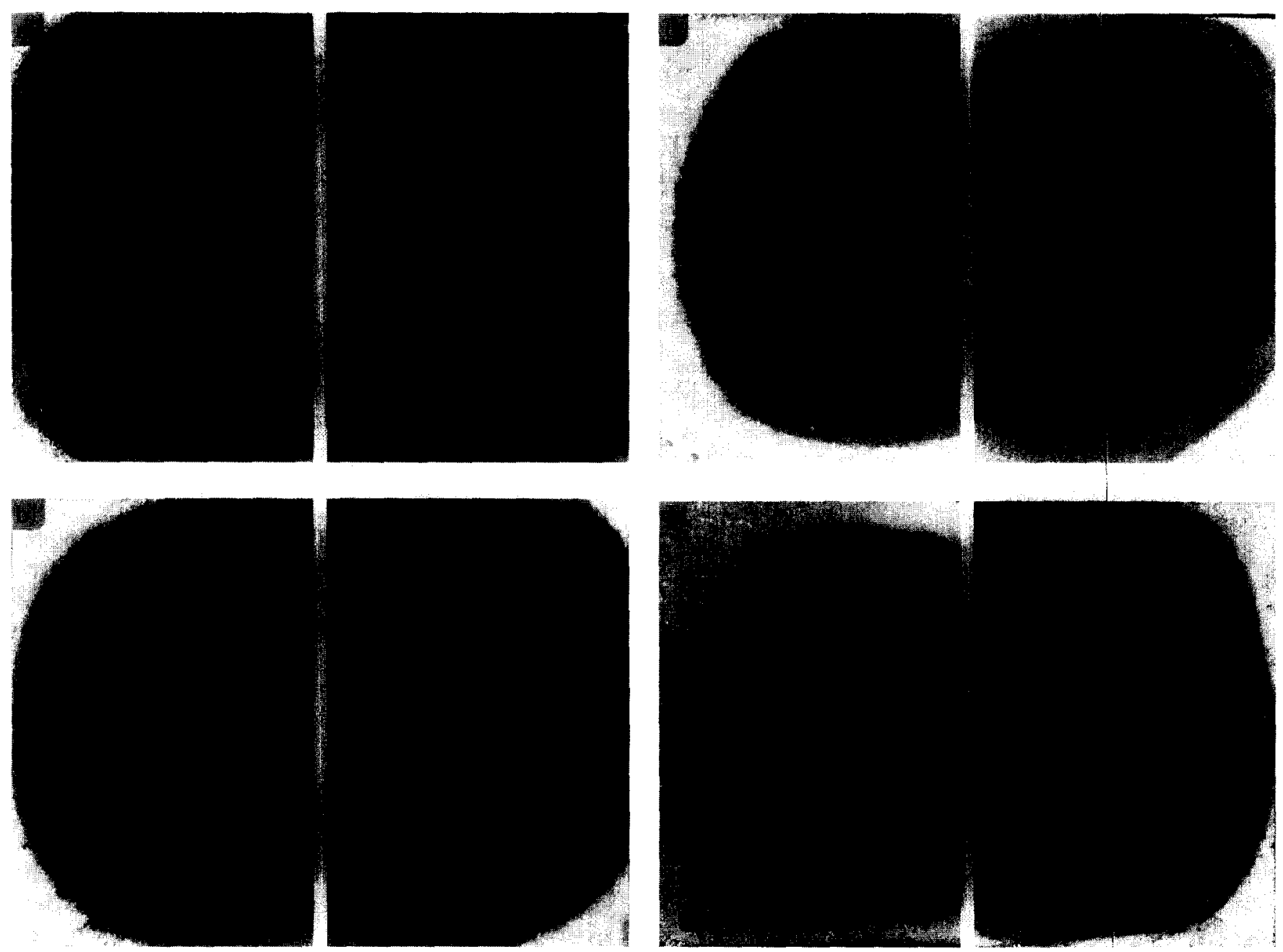

Fig. 6. BM images showing the change in large-scale morphology for diamond deposits obtained by turbulent (left-hand side) and laminar (right-hand side) flames, at distances of (a) $1 \mathrm{~mm}$, (b) $2 \mathrm{~mm}$, (c) $4 \mathrm{~mm}$ and (d) $6 \mathrm{~mm}$ from the tip of the flame cone. All photographs are on the same scale and reveal somewhat more than half of the samples.

to become oriented with their $\langle 100\rangle$ directions perpendicular to the substrate. At $d=6 \mathrm{~mm}$ the deposits in the central area reveal individual columnar crystallites with pyramidal tops corresponding to those of octahedra with $\{111\}$ faces. The absence of $\{100\}$ facets on these tops indicates that the growth rate in the [100] direction, which is almost perpendicular to the substrate, under these conditions is at least $3^{1 / 2}$ times larger than that of $\{111\}[33,34]$. Finally, at $d=8 \mathrm{~mm}$, where the annulus of isolated crystallites has almost reached the middle of the samples, the deposits reveal a thin amorphous layer of ball-like features quite similar to those shown for the laminar specimen at the right-hand sides of Figs. 7(c) and (d).

For the core 7 ne arising in the middle of the laminar samples at $d \geqslant 2.4 \mathrm{~mm}$, a similar change in morphology as described for the central areas of turbulent samples takes place, but at much smaller distances from the flame front. The right-hand side of Fig. 7(a) shows that at $d=2 \mathrm{~mm}$ this area is already dominated by large
$\{100\}$ faces. At $2.4 \mathrm{~mm}$ the continuous layer has broken up into individual columnar crystallites with very small $\{100\}$ top faces almost parallel to the substrate. The $\{100\}$ faces have disappeared at $2.8 \mathrm{~mm}$ (Fig. 7(b)), leading to a crystallite morphology as described for the turbulent layer grown at $d=6 \mathrm{~mm}$. In the case of the core zone however, the crystallites are embedded in a kind of amorphous layer. For $d \geqslant 4 \mathrm{~mm}$ these core zones do not show crystallites, but are fully covered by an amorphous layer less than $5 \mu \mathrm{m}$ thick with ball-like features (see right-hand sides of Figs. 7(c) and (d)). The central area of the laminar deposits between the core zones and the annulus with isolated crystallites changes in the same way, but more slowly. Although the crystallites are highly deteriorated, this part of the central area still does not form a complete amorphous layer for $d=$ $10 \mathrm{~mm}$. The crystallites deposited outside the annulus are well shaped for all values of $d$. This indicates that contradictory reports in literature between diamond deposition in the centre $[6,14]$ and in a circular area 

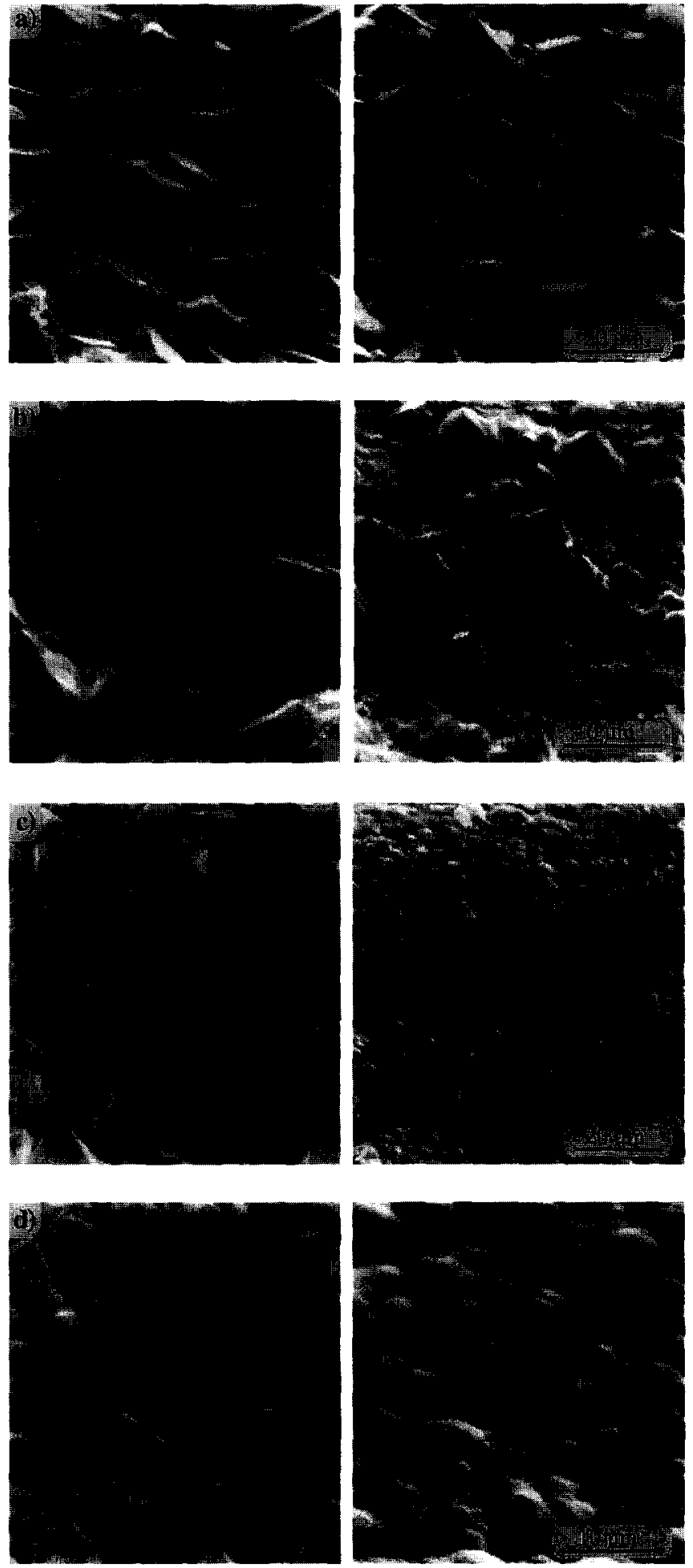

Fig. 7. SEM images showing the detailed morphology of turbulent (left-hand side) and laminar (right-hand side) diamond deposits grown at distances of (a) $2 \mathrm{~mm}$, (b) $2.8 \mathrm{~mm}$, (c) $4 \mathrm{~mm}$ and (d) $6 \mathrm{~mm}$ from the tip of the flame cone. Left- and right-hand sides of the photographs are on the same scale.

around the centre [35] can be explained by a difference in the applied substrate to flame cone distance.

Summarizing, relatively close to the flame front the central areas of the flame-grown deposits reveal ran- domly oriented crystallites with $\{111\}$ as well as $\{100\}$ faces. Further away from the flame front the $\{100\}$ faces align more and more parallel to the substrate surface and at the same time their relative dimensions, i.e. their morphological importance, decrease. Simultaneous with this process the $\{111\}$ faces suffer from an increasing deterioration due to the occurrence of twinning and stacking faults. Finally, at very large distances, where the gas phase activation is low, an amorphous rather than a crystalline layer is obtained. This change in morphology as a function of the substrate distance to the flame front proceeds fastest for the core zone arising at the laminar samples, slowest for the central area around this zone and at an intermediate speed for the central areas of the turbulent samples.

Raman measurements for the core zones on the laminar deposits grown at $d \geqslant 2 \mathrm{~mm}$ reveal a decreasing diamond phonon line and an increased signal of the non-diamond carbon phases compared with the central area directly around it. Laser-induced fluorescence measurements in a laminar flame reveal that the described phenomena are related to a local minimum of the $\mathrm{CH}$ and $\mathrm{C}_{2}$ concentrations in the gas phase directly above the core zone $[35,36]$. Possibly this is caused by the onset of soot formation in the gas phase further away from the activation zone of the flame [2]. This probably proceeds via a polymerization reaction to aromatic hydrocarbons involving $C_{2}$ [35]. Upon deposition these large molecules are most easily built into the diamond via the $\{111\}$ faces, which have a graphite-like surface structure, leading to a deterioration of these facets. The left-hand side of Fig. 7(d) shows that close to the top of the columnar crystallites, i.e. shortly after nucleation, the $\{111\}$ facets are still flat, but at positions further from the top, after prolonged exposure to the gas phase, they are already completely deteriorated. With increasing concentrations of the large molecules at larger distances from the flame front the crystallites become affected closer to the top, finally resulting in the deposition of a non-diamond carbon layer on the substrate.

\section{Discussion}

The growth results obtained with different supersaturations agree well with those of a former comparison between turbulent and laminar flames reported by Alers et al. [9], although the relatively low growth rates of $30 \mu \mathrm{m} \mathrm{h}^{-1}$ for laminar flames, as obtained by them, were generally not observed in the present work. This, however, might be due to small differences in the position of the substrate in the flame, which were estimated by the naked eye at that time. Another explanation might be the use of lower deposition temperatures or smaller burners, with exit openings of $0.8 \mathrm{~mm}$ in diameter, than 
in the present study. Smaller burners with an exit opening of $1.0 \mathrm{~mm}$ in diameter were also used in a previous study on the influence of $S_{\mathrm{ac}}$ and $d$ on samples grown by laminar flames [6]. The linear increase in growth rate with $S_{\mathrm{ac}}$, the sharp decrease of it with increasing $d$ close to the flame front and even the breaking up of the central area for $S_{\mathrm{ac}} \geqslant 8 \%$ and small values of $d$, as reported in the present work, were also encountered at that time.

Most observed differences between diamond layers grown by the two types of flame can be well understood in terms of the higher mixing rates of mass and heat in the gas phase of the turbulent flame compared with the laminar one. For example, the core zone arising in the middle of deposits grown with a laminar flame at large distances from the flame front does not arise for the turbulent samples, since the gas phase directly above this area is mixed with that around it. In the same way, the smaller central area of the turbulent specimen can be explained by a larger radial mass transfer of activated nitrogen into the turbulent gas phase. In the following a model is presented to describe the observed differences in growth rate between the turbulent and laminar flames as a function of $d$ and $S_{\text {ac }}$.

Fig. 5(b) shows that for $f_{\mathrm{ux}}=3 \mathrm{slm}$ and $S_{\mathrm{ac}}=5 \%$ the growth rates increase rapidly with decreasing values for $d$. Assuming that the growth rate is determined by the amount of growth precursors in the gas phase, this indicates the presence of a high concentration of hydrocarbon radicals suited for diamond deposition [R.] close to the flame front. Due to a temperature gradient and gas phase reactions between hydrocarbon radicals, the concentration of these specific species decreases rapidly with distance until some kind of equilibrium concentration $\left[\mathrm{R}_{\mathrm{eq}}{ }^{\circ}\right]$ is reached at approximately $1 \mathrm{~mm}$ from the flame front. Just like the primary combustion at the flame front, this process is expected to proceed more gradually in the turbulent flame due to the high mixing rates. At the outer boundary of the acetylene feather all hydrocarbons, which cause the luminescence of the feather [2], have reacted with oxygen from the ambient and are converted into more stable species like $\mathrm{CO}$ or $\mathrm{CO}_{2}$. Again, due to the larger rates of mass transfer, the oxygen penetrates more easily into the turbulent feather. The oxygen reacts with $\mathbf{R}$, reducing its concentration by an amount of $\left[\mathrm{R}_{\mathrm{ox}}{ }^{\cdot}\right]$ compared with the equilibrium level. At a certain distance from the flame front this causes a decrease in growth rate compared with that obtained by the laminar flame, for which the equilibrium level is maintained longer (Fig. 5). The process proceeds gradually until it goes to completion sooner than for the laminar flame, as is indicated by the smaller feather of the free-burning turbulent flame $\lceil 10\rceil$. It can be expected that this quantitative description of the gas phase is also valid for other values of $S_{\mathrm{ac}}$.

In a first-order approximation the amount of $[R \cdot]$ created at the flame front can be regarded as proportional to $S_{\mathrm{ac}}$. Due to the reactions between the radicals, $[R \cdot]$ is assumed to decrease exponentially until the equilibrium concentration $\left[\mathrm{R}_{\mathrm{eq}}{ }^{\cdot}\right]$ is reached, which is also proportional to $S_{\mathrm{ac}}$. In addition, the decrease in $\mathrm{R}$, due to the reaction with oxygen, can be assumed to proceed exponentially as the amount of available oxygen diffused into the feather increases with the distance from the flame front, until $[R \cdot]=0$ close to the outer boundary of the feather. The overall value of $[R \cdot]$ as a function of $d$ is obtained by the superposition of these two processes. Fig. 8 shows the described approach with the two subprocesses (dashed lines) and the overall value fitted to the growth rates of the turbulent samples with $S_{\mathrm{ac}}=5 \%$. For the turbulent case, $[\mathrm{R} \cdot]$ as a function of $d$ for supersaturations of $2.5 \%$ and $7.5 \%$ is obtained by shifting the curves representing the two sub-processes up or down with the equilibrium level and in such a way in the horizontal direction that the boundary conditions given by the assumptions stated above are fulfilled. Compared with the turbulent situation, [R·] in the laminar gas phase is much closer to the equilibrium concentration because of the lower mixing ratcs. Fig. 8 indicates that at $d=1.5 \mathrm{~mm},[\mathrm{R} \cdot]$ in the turbulent flame is less than the equilibrium level, and thus less than [R.] in the laminar flame for $S_{\mathrm{ac}}=2.5 \%$, but exceeds this level more and more for higher values of $S_{\mathrm{ac}}$. Quantitatively this agrees well with the results of the growth experiments shown in Fig. 2 with the crossing of the laminar and turbulent lines. Although this description provides a way to explain the observed differences in diamond deposition between turbulent and laminar flames, it is only a rough model based on several assumptions concerning the gas phase which cannot be verified by deposition experiments. A detailed description of the gas phasc above the growing surface requires examination by an in situ technique like laser-induced fluorescence [36].

In accordance with the results of the CL measurements, homoepitaxially grown diamond single crystals which were positioned within a few millimetres from the flame front along the central axis of the acetylene feather show virtually no incorporation of nitrogen as measured by infrared spectroscopy $[4,5]$ and photoluminescence [8]. In situ mass spectroscopic measurements reveal the presence of relatively large mole fractions of about 0.1 $N_{2}$ in the same region of the feather [26,37]. This indicates that only a small part of the nitrogen in the gas phase is activated and/or there is a very low incorporation efficiency of $\mathrm{N}$ into the diamond lattice as reported in literature [20]. Surprisingly, the mass spectrometric experiments reveal that the nitrogen concentration close to the flame front is the same for turbulent and laminar flames. In contrast with the model presented in this work, this implies that the introduction of turbulence does not enhance the entrainment of air from the 


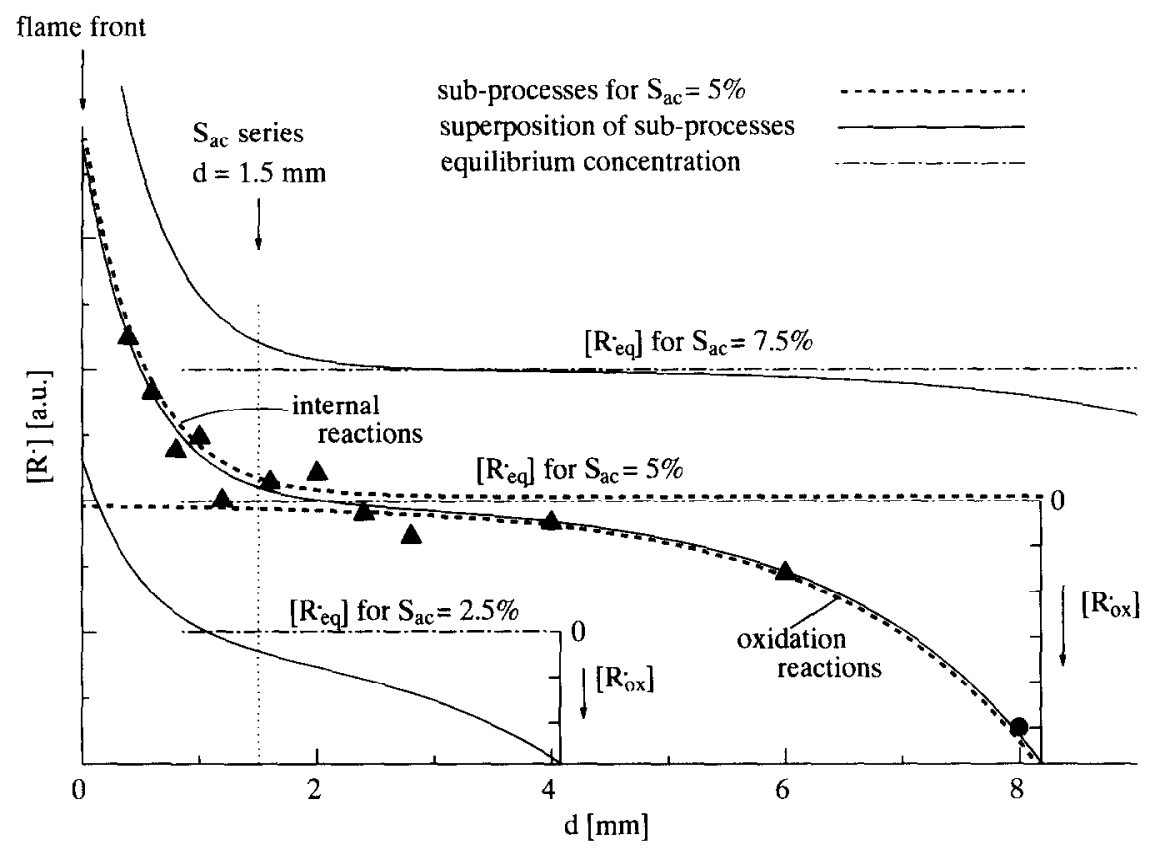

Fig. 8. [R-] as a function $d$ for different values of $S_{\text {ac }}$ according to the model described in the text. The markers represent the combined data for the turbulent flame as shown in Figs. 5(a) and (b).

ambient. However, in the mass spectroscopic study a commercially available burner was used and turbulence was achieved using much higher gas flows compared with the laminar situation, which counterbalances the effect of enhanced air entrainment. Furthermore, photographs of such burners operating in the turbulent regime show that the top of the conical flame front has become blurred, but that it is still well defined along the sides, like a laminar flame [5]. In contrast with this, almost the entire flame front of the turbulent flame used in the present study is blurred and is much shorter relative to the exit opening of the burner [10]. This indicates a higher degree of turbulence, which probably causes the observed differences in diamond growth compared with the laminar flame.

\section{Conclusions}

In this study it has been demonstrated that, depending on the supersaturation and substrate distance to the flame front, the growth rate obtained by a turbulent flame can be higher as well as lower than for a laminar flame. These differences are explained by a model involving the higher mixing rates in the turbulent gas phase. The morphological differences observed between samples grown by both flame types can also be explained by the larger mass transport rates in the turbulent flame. At a deposition temperature of $1100{ }^{\circ} \mathrm{C}$ and an oxygen flow of $3 \mathrm{slm}$, continuous layers of connected crystallites could be grown at rates exceeding $75 \mu \mathrm{m} \mathrm{h}^{-1}$ with both flame types. In general the diamond peak in the Raman spectra show a higher peak to background ratio for samples grown by the turbulent flame. However, in spite of the more rounded shape of the turbulent flame front, the homogeneous central area for turbulent deposits is smaller than for laminar specimens. Therefore it can be concluded that, depending on the desired results, the use of both flame types at different growth conditions can be advantageous.

It was shown that contradictory reports between diamond deposition in the centre and deposition in a circular area around the centre are related to different flame cone to substrate distances used in the experiments. The presence of high concentrations of large hydrocarbon fractions relatively far away from the flame front, i.e. the activation zone, probably results in the deterioration of the $\{111\}$ faces in the centre of the deposits, finally leading to the deposition of an amorphous carbon layer. Growth of columnar crystallites with the $\langle 100\rangle$ directions perpendicular to the substrate, as obtained by both flame types, is also accompanied by such a deterioration of the growth via the $\{111\}$ faces. The incorporation of nitrogen can be responsible for a similar process, which appears in the annulus of isolated crystallites directly around the central area.

\section{Acknowledgements}

The authors wish to thank Mr F. Derksen, Mr W. Corbeek and Mr W. Schut for their help in the continuous improvement of the flame growth equipment. Mr J. Spaanjaars, Mr Klein-Douwel and Dr G. Janssen 
are acknowledged for useful discussions. This work was financially supported by the European Community Brite Euram project BE 5099-92 under contract BRE-2-0147.

\section{References}

[1] Y. Hirose and N. Kondo, Extended Abstracts, 35th Jpn. Appl. Phys. Spring Meeting, March 1988, p. 434.

[2] A.G. Gaydon and H.G. Wolfhard, Flames: Their Structure, Radiation and Temperature, Chapman \& Hall, London, 1979.

[3] K.A. Snail and L.M. Hanssen, J. Cryst Growth., 112 (1991) 651.

[4] J.J. Schermer, W.J.P. van Enckevort and L.J. Giling, Diamond Relat. Mater., 3 (1994) 408.

[5] K.A. Snail, R.G. Vardiman, J.P. Estrera, J.W. Glesener, C. Merzbacher, C.J. Craigie, C.M. Marks, R. Glosser and J.A. Freitas Jr., J. Appl. Phys., 74 (1993) 7561.

[6] J.J. Schermer, J.E.M. Hogenkamp, G.C.J. Otter, G. Janssen, W.J.P. van Enckevort and L.J. Giling, Diamond Relat. Mater., 2 (1993) 1149

[7] K.A. Snail and C.J. Craigie, Appl. Phys. Lett., 58 (1991) 1875.

[8] K.A. Snail, C.L. Vold, C.M. Marks and J.A. Freitas Jr., Diamond Relat. Mater., 1 (1992) 180.

[9] P. Alers, W. Hänni and H.E. Hintermann, Diamond Relat. Mater., 2 (1993) 393.

[10] J.J. Schermer, P. Alers and L.J. Giling, J. Appl. Phys., in press.

[11] Y. Hirose, S. Amanuma and K. Komaki, J. Appl. Phys., 68 (1990) 6401.

[12] L.M. Hanssen, K.A. Snail, W.A. Carrington, J.E. Butler, S. Kellogg and D.B. Oakes, Thin Solid Films, 196 (1991) 271.

[13] W.J.P. van Enckevort, G. Janssen, W. Vollenberg, J.J. Schermer and L.J. Giling, Diamond Relat. Mater., 2 (1993) 997.

[14] K.V. Ravi, J. Mater. Res., 7 (1992) 384.

[15] P.J. Dean, Phys. Rev., 139 (1965) A588.

[16] J.J. Dubray, W.A. Yarbrough and C.G. Pantano in R.E. Clausing et al. (eds.), Diamond and Diamond-Like Films and Coatings, Plenum, New York, 1991.

[17] S. Lijima, Y. Aikawa and K. Baba, Appl. Phys. Lett., 57 (1990) 2646

[18] L.H. Robins, L.P. Cook, E.N. Farabaugh and A. Feldman, Phys. Rev. B, 39 (1989) 13367.

[19] A.T. Collins, M. Kamo and Y. Sato, J. Phys. D, 22 (1989) 1402.

[20] S. Jin and T.D. Moustakas, Appl. Phys. Lett., 65 (1994) 403.

[21] G.Z. Cao, W.J.P van Enckevort, L.J. Giling and R.C.M. de Kruif, Appl. Phys. Lett., 66 (1995) 688.

[22] G.Z. Cao, J.J. Schermer, W.J.P van Enckevort, W.A.L.M. Elst and L.J. Giling, in preparation.

[23] C.J. Ammerlaan, Inst. Phys. Conf. Ser., 59 (1981) 81.

[24] L.J. Giling and W.J.P. van Enckevort, Surf. Sci., 161 (1985) 567.

[25] J.J. Schermer, W.J.P. van Enckevort and L.J. Giling, J. Cryst. Growth, 148 (1995) 248.

[26] N.A. Pangarov, in N.N. Sheftal (ed.), Rost Kristallov, Vol. 10, Nauka, Moscow, 1974.

[27] K.V. Ravi, C.A. Koch, H.S. Hu and A. Joshi, J. Mater. Res., 5 (1990) 2356.

[28] C.M. Marks, H.R. Burris, J. Grun and K.A. Snail, J. Appl. Phys., 73 (1993) 755 .

[29] R. Haubner and B. Lux, Diamond Films Technol., 3 (1994) 209.

[30] H.M. Grimditch, E. Anastassakis and M. Cardona, Phys. Rev. B, 18 (1978) 901.

[31] M. Yoshikawa, G. Katagiri, H. Ishida, A. Ishitani, M. Ono and K. Matsumara, Appl. Phys. Lett., 55 (1989) 2608.

$\lceil 32\rceil$ G. Janssen, Homoepitaxial diamond synthesized by CVD processes, Thesis, University of Nijmegen, 1994.

[33] B.V. Spitsyn, L.L. Bouilov and B.V. Derjaguin, J. Cryst. Growth. 52 (1981) 219

[34] C. Wild, P. Koidl, W. Müller-Sebert, H. Walcher, R. Kohl, N. Herres, R. Locher, R. Samlenski and R. Brenn, Diamond Relat. Mater., 2 (1993) 491.

[35] M.A. Cappelli and P.H. Paul, J. Appl. Phys., 67 (1990) 2596.

[36] R. Klein-Douwel, J. Spaanjaars and J.J. ter Meulen, J. Appl. Phys., in press.

[37] Y. Matsui, A. Yuuki, M. Sahara and Y. Hirose, Jpn. J. Appl. Phys., 28 (1989) 1718 